Ann. Zootech., I978, 27 (2), I59-I67.

\title{
Estimation de la composition chimique du corps entier des bovins à partir du poids des dépôts adipeux totaux
}

\author{
J. ROBELIN et Y. GEAY \\ avec la collaboration technique \\ de Christiane BARBOIRON et de Roland JAI, F, R \\ Laboratoive de la Production de Viande, \\ Centre de Recherches de Clermont-Ferrand, I.N.R.A., \\ Theix, 63II0 Beaumont (France)
}

\section{Résumé}

Cette étude propose des équations permettant d'estimer le poids des protéines, des lipides et le contenu en énergie du corps entier des bovins, et pas seulement de la carcasse, à partir du poids vif vide (poids vif - poids du contenu digestif) et du poids des dépôts adipeux totaux (de la carcasse et du cinquième quartier). Ces équations ont été calculées à partir de la mesure directe par broyage et analyse chimique du corps entier de 84 bovins.

Les coefficients de variation résiduelle (écart-type de la régression exprimé en $p$. cent de la variable dépendante) sont relativement faibles $(6,5$ p. cent; 2,8 p. cent et 3,6 p. cent respectivement pour les lipides, les protéines et l'énergie). La relation allonétrique entre la masse délipidée (poids vif vide - poids des lipides) et le poids de protéines est indépendante de la race et du sexe des animaux; elle est par ailleurs très voisine de celle qui est observée à partir de résultats bibliographiques (fig. I).

La précision de la méthode d'estimation proposée est discutée par rapport à la précision de la mesure directe de la composition chimique du corps entier.

\section{Introduction}

La détermination des besoins physiologiques des animaux en croissance, ainsi que 1a mesure de la valeur nutritive des aliments, nécessitent la connaissance de la composition du corps entier des animaux à différents stades afin de calculer les quantités de protéines et d'énergie fixée dans le croît. L'estimation par la méthode des espaces de diffusion de l'eau lourde (ROBELIN, I977) est intéressante lorsqu'on souhaite maintenir les animaux en vie. Son coût ne justifie plus son emploi lorsque les animaux doivent être abattus. Il est alors souhaitable, pour des raisons économiques évidentes, de disposer d'une méthode rapide et précise d'estimation de la composition du corps entier qui permettrait d'éviter le broyage et l'analyse chimique de ce dernier. 
Les méthodes proposées jusqu'à présent, reposant sur des critères faciles a mesurer à l'abattage (HopPER, I944; RoBELIN, GEAY et BÉRANGER, I975 a) ne permettent d'estimer que la composition de la carcasse. Or, celle-ci ne représente que 55 à $70 \mathrm{p}$. cent de la masse corporelle totale. On pourrait déduire la composition du corps entier de celle de la carcasse comme le proposent GaRRET'T et HINMAN (I G69). Toutefois, ce calcul ne peut être généralisé que si la relation entre la composition de la carcasse et celle du cinquième quartier est constante quels que soient la race et le sexe des animaux. Cette hypothèse ne peut probablement pas être retenue compte tenu de la variabilité dans la répartition des dépôts adipeux entre la carcasse et le cinquième quartier (BÉRANGER et RoBELIN, I977). C'est pourquoi, il nous est apparu nécessaire de rechercher une méthode d'estimation de la composition du corps entier qui tienne compte de cette variabilité, et qui s'appuie par conséquent d'une part sur des critères reflétant la composition de la carcasse, et sur des critères reflétant celle du cinquième quartier d'autre part. Cette méthode permet, grâce à des relations successives, d'estimer de proche en proche les différents composants chimiques du corps entier : lipides puis protéines, eau et énergie fixée.

\section{Matériel et méthodes}

Quatre-vingt-quatre bovins âgés de 3 à Ig mois dont le poids vif variait entre I 20 et 7 Io $\mathrm{kg}$ ont été utilisés. Leur demi-carcasse droite a été disséquée. Nous avons mesuré, après dissection complète, le poids des dépôts adipeux de la demi-carcasse droite. Ce poids a ensuite été corrigé pour le rapporter à la totalité de la carcasse, et pour tenir compte de la perte d'eau entre l'abattage et la dissection. I,e poids considéré des dépôts adipeux de la carcasse est égal au poids observé après dissection multiplié par le rapport $\mathrm{B} / \mathrm{A}(\mathrm{A}=$ somme des poids des éléments de la demicarcasse après dissection; $\mathrm{B}=$ poids de la carcasse chaude entière après abattage). Les poids des dépôts adipeux du cinquième quartier (péritonéaux, périrénaux, mésentériques et péricardiaques) ont été mesurés immédiatement après l'abattage. Le poids des dépôts adipeux totaux que nous avons utilisé est la somme des dépôts adipeux du cinquième quartier et des dépôts de la carcasse (après correction).

La demi-carcasse droite ainsi que la totalité du cinquième quartier ont été broyées et analysées (eau, lipides, protéines, énergie) selon la méthode décrite précédemment (Robelin, Geay, BÉRANGER, I975 b).

Afin d'estimer la composition du corps entier, nous avons établi des relations entre certains de ses constituants anatomiques et ses constituants chimiques au cours de 3 étapes successives.

I. Estimation du poids des lipides corporels à partir du poids des dépôts adipeux totaux. Cette estimation repose sur le fait que la teneur en lipides des dépôts adipeux comme des autres tissus angmente en relation étroite avec le poids de ces dépôts.

2. Estimation du poids des protéines et d'eau à partir de la masse délipidée. Depuis les travaux de Moulton, Trowbridge et HaIgh (I922), de nombrenx auteurs (CAILOW, I947; REID, WELIINGTON et DUNN, I955; LOHMAN, I97 I) ont montré que la masse délipidée (masse corporelle - lipides) avait une composition pratiquement constante en protéines et eau, après un stade physiologique appelé par Moul'Ton " maturité chimique " et qui serait situé à 4 p. cent environ de la 
durée de vie de l'animal. REID, WELLINGTON et DUNN (I955) ont toutefois constaté une légère évolution de cette composition avec l'âge des animaux. C'est pour tenir compte de cette évolution que nous avons recherché une relation entre les protéines ou l'eau d'une part et la masse délipidée d'autre part.

3. Estimation de la quantité d'énergie du corps entier à partir du poids vif vide et des dépôts adipeux totaux ou calcul à partir de la relation:

Énergie $($ Mcal $)=5,4^{8} \times$ protéines $(\mathrm{kg})+9,37$ matières grasses $(\mathrm{kg})$.

Les coefficients 5,48 et 9,37 ont été obtenus à partir de mesures calorimétriques effectuées sur 80 carcasses (ROBELIN et GEAY, I976 b). Ils sont très voisins de ceux que FERREI $L_{1}$, GarReTT et HINMAN (I976) ont obtenus récemment.

L'analyse statistique des résultats a été effectuée après transformation logarithmique des variables, de façon à se rapprocher des conditions de normalité auxquelles font appel les tests utilisés. Cette transformation a pour effet de rendre la variabilité des différentes mesures moins dépendantes de la valeur prise par ces variables. Elle permet également d'exprimer l'évolution de ces variables selon la relation allométrique classique.

\section{Résultats et discussion}

Relation entre les lipides et les dépôts adipeux totaux

La relation obtenue entre le poids des lipides $(L, k g)$ et celui des dépôts adipeux totaux (DAT, $\mathrm{kg}$ ) a été la suivante :

$$
\mathrm{L}=\mathrm{I}, \mathrm{I} 346 \times \operatorname{DAT}^{0,992} \quad \mathrm{R}^{2}=0,99 \mathrm{I} \quad \mathrm{CVR}=6,5 \text { p. cent de } \mathrm{I}_{1}
$$

Les variations dans le poids des dépôts adipeux rendent compte de 99 p. cent de la variabilité dans le poids des lipides. Alors que la variabilité d'ordre biologique dans le poids des lipides est de I 9 , I p. cent à même poids vif vide, elle est réduite à 6,5 p. cent seulement lorsque le poids des dépôts adipeux est fixé. Cette relation étroite entre les dépôts adipeux et les lipides n'est pas surprenante; elle a déjà été mentionnée par CALLOW (I948) et par nous-mêmes (ROBEIIN, GEAY et BÉRANGER, I 975 b). L'exposant 0,992 montre que le poids des lipides est pratiquement proportionnel à celui des dépôts adipeux.

L'analyse de variance sur les écarts à la régression n'a pas permis de mettre en évidence un effet significatif de la race et de l'âge des animaux sur la relation. Nous n'avons pas non plus mis en évidence d'effet significatif du sexe; cependant, le nombre limité de femelles utilisées dans l'analyse en est peut-être la cause.

Il est important de rappeler que l'estimation du poids des lipides n'est correcte, que si le poids des dépôts adipeux totaux, utilisé dans la relation, correspond exactement à celui que nous avons défini précédemment (cf. Matériel et Méthodes). Ainsi, appliquée aux résultats de Schuiz, Oslage et Dafnicke (I974) sur 44 animaux, cette équation surestime de 2 à 4 p. cent le poids réel des lipides mesuré par analyse. Cela peut être dî au fait que ces auteurs n'avaient pas inclu les dépôts adipeux péricardiaques qui représentent 2 à 4 p. cent de la masse adipeuse totale.

Avant d'utiliser cette relation, il serait donc nécessaire de lui appliquer un facteur de correction dépendant des conditions expérimentales de mesure du poids 


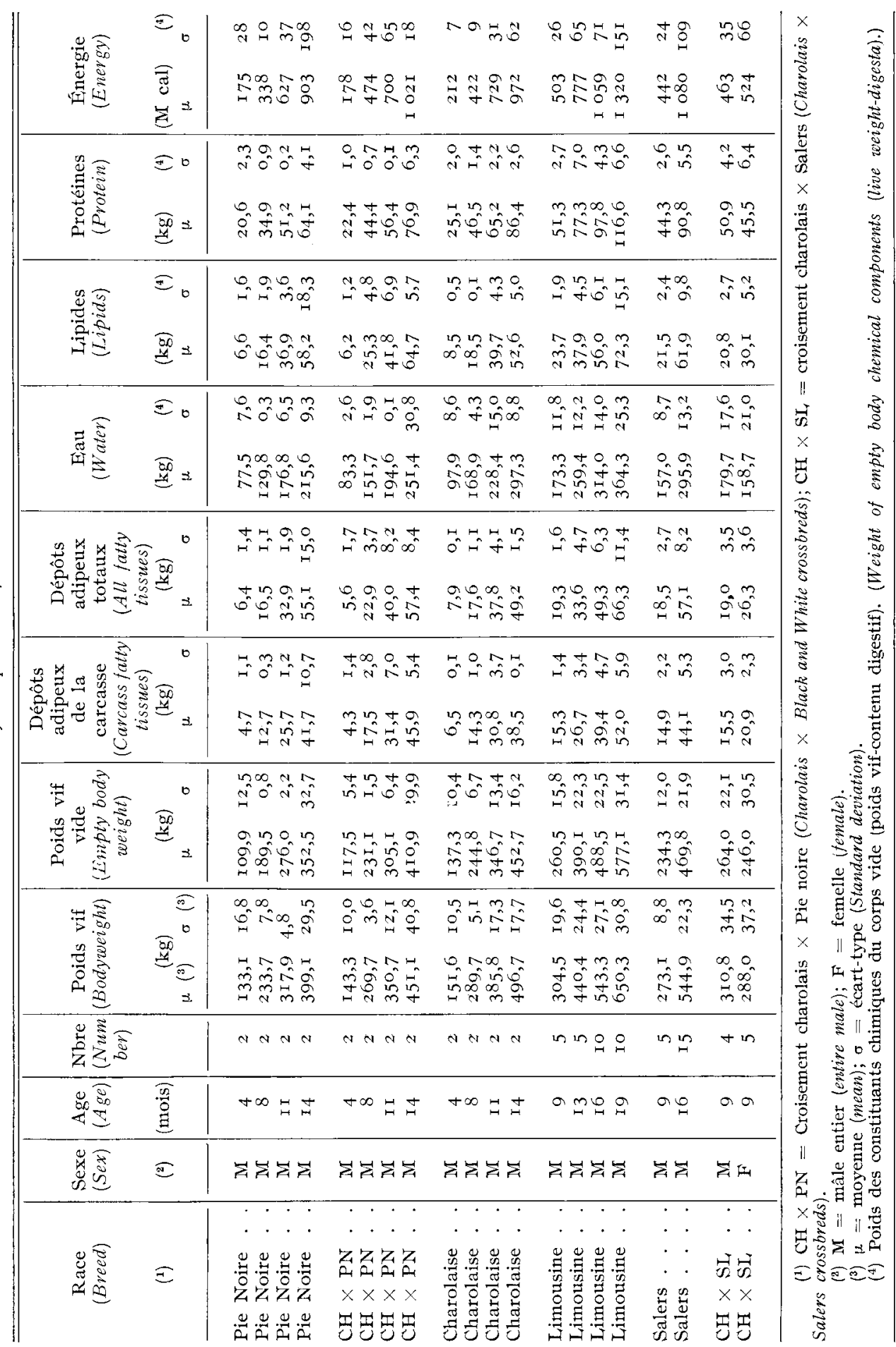


des dépôts adipeux. Ce facteur de correction pourrait être simplement le rapport La/Le, La étant le poids moyen de lipides mesuré par analyse chimique du corps entier de quelques animaux (5 à Io), Le étant le poids moyen de lipides de ces mêmes animaux, estimé à partir de l'équation proposée précédemment.

\section{Relation entre les protéines ou l'eau et la masse délipidée}

Les relations obtenues entre les poids des protéines ( $P, \mathrm{~kg})$ d'eau $(\mathrm{H}, \mathrm{kg})$ et la masse délipidée ( $\mathrm{MI}), \mathrm{kg}=$ poids vif vide — poids des lipides) ont été les suivantes :

$$
\begin{array}{lll}
\mathrm{P}=0, \mathrm{I} 259 \times \mathrm{MD}^{1,096} & \mathrm{R}^{2}=0,996 & \mathrm{CVR}=2,8 \text { p. cent } \\
\mathrm{H}=0,8477 \times \mathrm{MD}^{0,974} & \mathrm{R}^{2}=0,999 & \mathrm{CVR}=\mathrm{I}, \mathrm{I} \text {. cent }
\end{array}
$$

Ces équations conduisent à des teneurs moyennes en protéines et en eau de la masse délipidée voisines de celles proposées par REII, WELI INGTON et DUNN (I955) pour les bovins. Cependant, l'ajustement de ces teneurs à la masse délipidée a permis de réduire sensiblement, dans le cas des protéines notamment, le coefficient de variation résiduelle par rapport aux valeurs obtenues par ces auteurs, même lorsque l'âge des animaux était pris en compte.

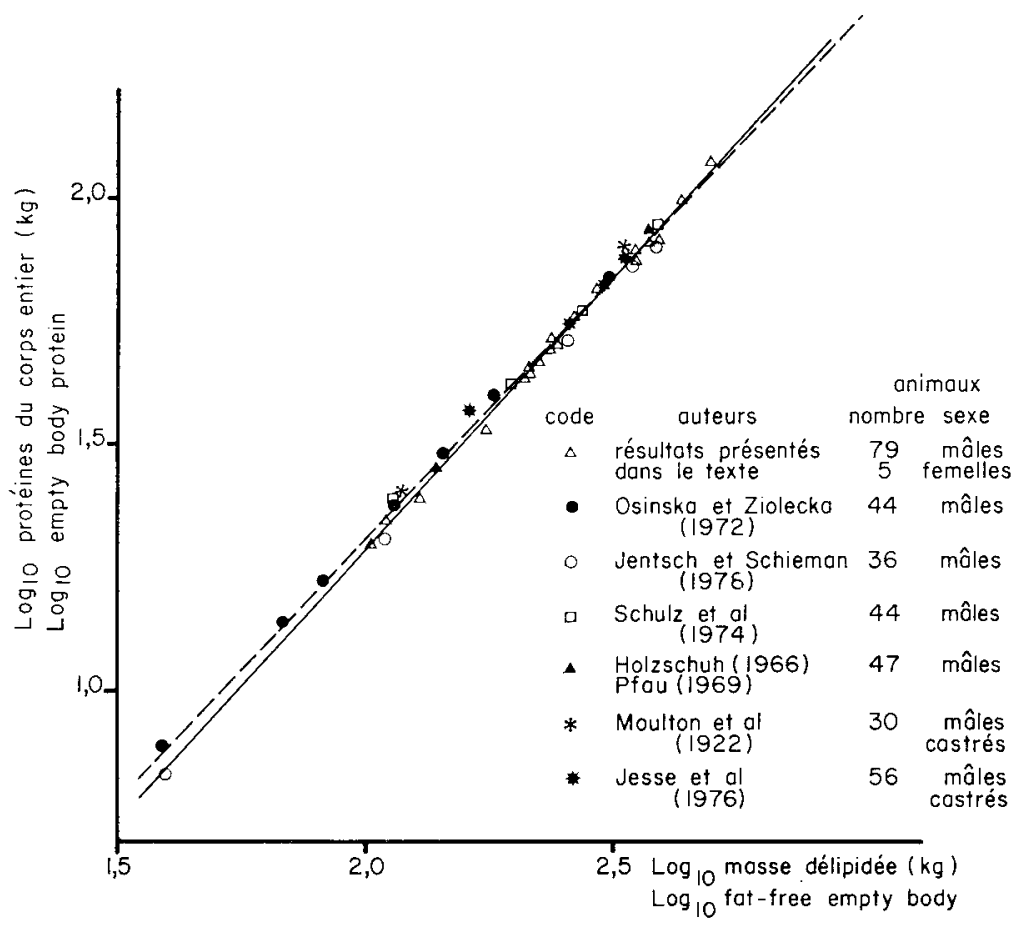

FIG. I. - Relation entre le poids des protéines du corps entier et la masse délipidée (poids vif vide - poids des lipides) chez les Bovins

Relationship between protein and lean body mass in cattle

(La ligne continue représente les résultats présentés dans le texte, la ligne discontinue l'ensemble des résultats de la bibliographie. The full line represents results presented in the text, the dotted line, data from the literature). 
Comme précédemment, l'analyse de variance des résidus n'a pas permis de mettre en évidence $11 n$ effet de la race ou du sexe des animaux sur la relation. Par ailleurs, d'après les résultats recueillis dans la bibliographie (fig. I), la relation entre les protéines et la masse délipidée semble pouvoir être appliquée à une variété d'animaux beaucoup plus large : mâles entiers ou castrés de races très précoces (Angus, Hereford) ou tardives (Charolaise, Limousine) et entre 40 et 7 Io $\mathrm{kg}$ de poids vif. Les relations calculées sur les moyennes de lots à partir de l'ensemble de ces résultats sont très voisines de celles que nous avons obtenues à partir de nos propres résultats :

$$
\begin{aligned}
& \mathrm{P}=0, \mathrm{I} 53 \mathrm{I} \times \text { M.D. }{ }^{1,0608} \quad \mathrm{CVR}=4, \mathrm{I} \text { p. cent } \\
& \mathrm{H}=0,7805 \times \text { M.D. }{ }^{0,9816} \quad \mathrm{CVR}=2,8 \text { p. cent }
\end{aligned}
$$

Cette constance à l'intérieur d'une espèce dans la relation entre les protéines et la masse délipidée est tout à fait comparable à celle qu'ont observée récemment BELL et STERN (I977) chez le rat entre des animaux normaux et des animaux génétiquement obèses. Par ailleurs ces relations sont comparables à celles qu'a obtenues DFLPECH (I966) chez, les oiseaux.

\section{Estimation $d u$ contenu en énergie ( $E, M$ cal) $d u$ corps entier}

L,a quantité d'énergie ( $\mathrm{E}, \mathrm{Mcal}$ ) est reliée au poids vif vide (P.V.V., kg) et au poids des dépôts adipeux totaux (D.A.T., kg) par la relation statistique suivante:

$$
\begin{aligned}
& \mathrm{E}=4, \text { III } \times \text { D.A.T. }{ }^{\mathbf{0}, 416} \times \text { P.V.V. }{ }^{\mathbf{0}, 633} \\
& \left(\mathrm{R}^{2}=0,996 ; \text { C.V.R. }=3,6 \text { p. cent de } \mathrm{E}\right)
\end{aligned}
$$

Lorsqu'on calcule $\mathrm{E}$ à partir des poids de protéines (Pe) et de lipides (L,e) estimés (cf. Matériel et Méthodes), le coefficient de variation résiduel est sensiblement plus élevé $(3,7 \mathrm{p}$. cent) que celui de la relation précédente. Toutefois, il nous semble plus logique d'utiliser l'équation : $\mathrm{E}=9,37 \times \mathrm{Le}+5,48 \times$ Pe qui correspond effectivement à la relation d'ordre physique entre l'énergie d'une part et le poids des protéines et de lipides d'autre part.

\section{Utilisation pratique de la méthode}

Connaissant le poids de l'ensemble des dépôts adipeux (de la carcasse et du cinquième quartier), il est donc possible d'estimer le poids des lipides, des protéines et la quantité d'énergie contenue dans le corps entier. L'intérêt pratique de cette méthode dépend en premier lieu de sa précision comparée à celle obtenue par broyage et analyse chimique du corps entier.

Nous avons effectué cette comparaison prenant comme exemple la détermination du contenu moyen en énergie d'un lot de $n$ bovins.

Dans le cas de la mesure directe (par broyage et analyse chimique), l'erreur attachée à la moyenne du lot est égale à la variabilité du contenu en énergie des animaux à même poids vif vide (6,8 p. cent; ROBEI,IN et GEAY, I976 a) divisé par $\sqrt{n}$.

Dans le cas de l'estimation à partir des dépôts adipeux totaux, on cumule cette première source de variation ( $\mathrm{VB}=6,8 \mathrm{p}$. cent) et l'erreur liée à l'estimation de l'énergie à partir des dépôts adipeux (cf. équation plus haut: $\mathrm{VE}=3,6 \mathrm{p}$. cent). L'ensemble de ces sources de variation, $\sqrt{\mathrm{VB}^{2}+\mathrm{VE}^{2} /} \sqrt{n}$ correspond à une erreur 
relative égale à $7,7 \mathrm{p}$. cent $/ \sqrt{n}$. Ainsi, on obtient la même erreur relative $3,0 \mathrm{p}$. cent en utilisant la méthode directe (broyage et analyse chimique) sur 5 animaux ou en procédant à l'estimation indirecte sur 7 animaux.

Dans la pratique, la méthode peut être simplifiée, dans la mesure où le poids des dépôts adipeux de la carcasse peut être estimé avec précision à partir de la composition de la II ête (ROBELIN, GEAY et BÉRANGER, I975 a). Cette estimation n'entraîne pas de biais sur la détermination du poids des lipides, et par suite des autres composants, mais elle se traduit par une diminution de sa précision. Dans l'exemple précédent, on doit ajouter à l'erreur calculée auparavant, une erreur $(\mathrm{VD}=3,4 \mathrm{p}$. cent) liée à l'estimation des dépôts adipeux de la carcasse, pondérée par le fait que les dépôts de la carcasse ne représentent que $80 \mathrm{p}$. cent des dépôts adipeux totaux, que ces dépôts interviennent à la puissance 0,42 dans la détermination de l'énergie. L'ensemble des sources de variation $\sqrt{\mathrm{VB}^{2}+\mathrm{VE}^{2}}$ $\overline{+\mathrm{VD}^{2}} / \sqrt{n}$ correspond alors à une erreur relative égale à $8,4 \mathrm{p}$. cent $/ \sqrt{n}$. Pour obtenir la même erreur relative $(3,0$ p. cent) que par broyage et analyse du corps entier de 5 animaux, il est alors nécessaire d'effectuer l'estimation indirecte sur un lot de 8 animaux. La comparaison de ces deux nombres montre bien l'intérêt de cette méthode d'estimation facile à mettre en œuvre et qui, si elle est appliquée sur un nombre suffisamment grand d'animaux, permet d'obtenir une précision très bonne sur la composition moyenne des animaux du lot.

En conclusion, l'originalité de la méthode proposée vient surtout du fait que, à partir de critères faciles à mesurer, elle permet d'estimer la composition du corps entier, et non seulement de la carcasse, ce qui était impossible avant, sinon par broyage et analyse chimique.

Par ailleurs, cette méthode est facile à mettre en ouvre et peu coûteuse; le poids des dépôts adipeux totaux peut être obtenu par la pesée des dépôts du cinquième quartier après l'abattage, et l'estimation indirecte des dépôts de la carcasse. Elle peut donc être appliquée à un grand nombre d'animaux. Enfin, le calcul précédent a montré que sa précision était très bonne par rapport à celle de la méthode directe par broyage du corps entier et analyse chimique.

Les coefficients des équations qui ont été proposés pourront bien sûr être affinés grâce aux mesures directes de la composition chimique des bovins que nous poursuivons; toutefois, compte tenu du nombre d'animaux déjà utilisés (84), de leur diversité et de la bonne concordance entre nos résultats et ceux de la bibliographie (fig. I), ces relations peuvent être utilisées avec un minimum de risque.

Accepté pour publication en décembre 1977.

\section{Summary}

\section{Estimation of the chemical composition of the bovine body from the total fatty tissue weight}

A set of equations is proposed for estimating the chemical composition of empty bovine bodies; these equations have been calculated from the results of chemical analyses of the body of 84 animals (Table I). The dependent variates are the empty body weight (PVV, kg; body weight - digesta), the weight of all fatty tissues (DAT, kg; carcass and fifth quarter fatty tissues), and the weight of the lean body mass (MD, kg; empty body weight - estimated weight of lipids). The main equations are:

- Lipids $(\mathrm{L} e, \mathrm{~kg})=\mathrm{I} . \mathrm{I} 346 \times \mathrm{DAT} 0.0992 \quad \mathrm{R}^{2}=0,99 \mathrm{I} \quad \mathrm{RCV}=6,5$ p. $\quad$ I00

( $\mathrm{RCV}$ is the residual coefficient of variation, expressed in $\%$ of the dependent variate). 
- Protein $\left(\mathrm{P}_{e}, \mathrm{~kg}\right)=0,1259 \times \mathrm{MD}^{1,096} \quad \mathrm{R}^{2}=0,996 \quad \mathrm{RCV}=2,8$ p. I00.

- Energy $\left(\mathrm{E}_{e}, \mathbf{M} \mathrm{cal}\right)=9,37 \times \mathrm{L}_{e}+5,48 \times \mathrm{P}_{e} \quad \mathrm{RCV}=3,7$ p. roo.

- Energy $\left(\mathrm{E}_{e}, \mathrm{Mcal}\right)=4, \mathrm{II} \times \mathrm{PVV}^{0,633} \times \mathrm{DAT}^{0,416} \quad \mathrm{R}^{2}=0,996 \quad \mathrm{RCV}=3,6$ p. Ioo.

The generalization of the first equation is discussed. According to figure $\mathrm{I}$, the relationship between protein and lean body mass seems to exist in a wide range of cattle types (entire males, castrated males, females, various breeds).

Using these equations, it is possible to estimate the chemical composition of cattle by weighing only the fifth quarter fatty tissues and by estimating carcass fatty tissues after dissection of a rib joint (RoBELIN, GEAY et BERANGER, I975a). The error connected with the determination of the mean energy content of the whole body of 8 animals is the same as that of the direct measurement of the energy content of 5 animals. This comparison clearly shows the interest of such a method for estimating the whole body composition of cattle after slaughter.

\section{Références bibliographiques}

BELL, G. E., SiERN J. S., 1977. Evaluation of body composition of young obese and lean zucker rats. Growth, 41, 63-80.

BERANGER C., ROBELIN J., I977. Influence du mode d'élevage, de la sélection et de l'alimentation sur l'état d'engraissement des bovins. Ann. Biol. anim. Bioch. Biophys. 17, 905-92I.

CALLOW E. H., 1947. Comparative studies of meat. I. The chemical composition of fatty and muscular tissue in relation to growth and fattening. $J$. A gric. Sci., 37, II3-I29.

CALLOW E. H., I948. Comparative studies of meat. II. The changes in the carcass during growth and fatteing, and their relation to the chemical composition of the fatty and muscular tissue. J. Agric. Sci., 38, I74-199.

DELPECI P., I966. Le poids frais délipidé chez Gallus gallus L. : relations qui unissent ses constituants. C. R. Acad. Se., 263, I 735-I 738 .

FERREI C. L., GarRetT W. N., Hinman N., I976. Estimation of body composition in pregnant and non pregnant heifers. J. Anim. Sci., 42, I I58-i 66.

GarretT W. N., Hinman N., I969. Re-evaluation of the relationship between carcass density and body composition of beef steers. J. Anim. Sci., 28, T-5.

HolzSCHuH W., I966. Wachstumversuch verbunden mit Körperanalysen bei Jungmastbullen zur Ermittlung des N-Ansatzes ans NPN-Verbindungen. Arch. Tierzucht, 9, I 59-1 76.

HOPPER T. H., I944. Methods of estimating the physical and chemical composition of cattle. J. Agric. Res., 68, 239-268.

Jentsch W., Schieman R., I976. Die Verwertung der Futterenergie durch wachsende Bullen. 2. Mitteilung. Arch. Tievernährung., 26, 519-532.

Jesse G. W., Thompson G. B., Chark J. L., Heprick H. B., Weimer K. G., I976. Fffects of ration energy and slaughter weight on composition of empty body and carcass gain of beef cattle. J. Anim. Sci., 43, 418-425.

Lohman T. G., I971. Biological variations in body composition. J. Anim. Sci., 32, 647-653.

Moulton C. R., Trow bridge P. F., HaIGH L. D, igz2. Studies in animal nutrition. III. Changes in chemical composition on different planes of nutrition. Mo. Agr. Expt. Station. Research. Bull. $\mathrm{n}^{\circ} 55$.

Osinska Z., Zrolecka A., 1972. A note on the protein content of the empty body of young Polisch Black and white Lowland bulls. Anim. Prod., 14, II9-I22.

Pfau A, 1969. Statistisch abgeleitete Relationen zur Bestimmung der grobchemischer Köperzusammensetzung beim Rind. $Z$. Tierzücht. Zücktungbiol., 85, 363-369.

REID J. T., WELLINGTON G. H., DUNN H. O., I955. Some relationships among the major chemical components of the bovine body and their application to nutritional investigations. J. Dairy Sci., 38, I 344-1 359 .

ROBELIN J., 1977. Estimation in vivo de la composition corporelle des agneaux à partir de l'espace de diffusion de l'eau lourde. Ann. Biol. Anim. Bioch. Biophys., 17, 95-105.

Robfin J., Geay Y., Beranger C., I975a. Estimation de la composition des carcasses de bovins mâles à partir de la composition d'un morceau monocostal prélevé au niveau de la I I ${ }^{\mathrm{e}}$ côte. I. Composition anatomique. Ann. Zootech., 24, 39I-402. 
ROBFIIN J., GEAX Y., BERANGLR C., I975b. Note : Estimation de la composition chimique des carcasses des jeunes bovins mâles, à partir de la proportion des dépôts adipeux d'un morceau monocostal prélevé au niveau de la i ic côte. Ann. Zootech., 24, 323-326.

RoI3Elin J., Gisy Y., I976a. Changes with age (9, I3, I6, I9 months) of protein and energy retention, and energy utilization by growing Limousin bulls. In Proc. 7 th Symp. on Energy Metabolism. E.A.A.P., 19, 2 I 3 -2 6 .

Romerin J., GEAY Y., I976b. Estimation de la composition des carcasses de jeunes bovins à partir de la composition d'un morceau monocostal prélevé au niveau de la i c côte. II. Composition chimique. Ann. Zootech, 25, 259-272.

Scmulz E., Oslagi: H. J., DAFNICkF; R., I974. Untersuchungen ïber die Zusammensetzung der Körpersubstanz sovie den Stoff und Energieansatz bei wachsenden Mastbullen. Fortschritte in der Tierphysiol. und Tierernährung., 4. 